\title{
Perinatal psychiatry: a new specialty or everyone's business?
}

\author{
Jacqueline Humphreys, Janet Obeney-Williams, Rachael W. Cheung \& Nisha Shah
}

\begin{abstract}
SUMMARY
Perinatal psychiatry is a relatively new subspecialty and controversy exists about such specialist provision. Differences can occur in how mental illnesses present in pregnancy, and there is a need to take into account both mother and baby. The risks of not treating perinatal mental illness can be both acute and chronic, and suicide in the context of untreated illness remains a leading indirect cause of maternal mortality. Despite the government's agenda of preventive healthcare, service provision is inequitable across the UK. Advice regarding treatment continues to be complex, and perinatal psychiatrists need to keep abreast of a growing evidence base. This review offers an overview of some current issues in the care of patients in the perinatal period and shows how specialised perinatal services are uniquely placed to meet their needs. Hopefully, it will prove useful to all clinicians responsible for the perinatal care of women and their families.
\end{abstract}

\section{LEARNING OBJECTIVES}

- Acquire increased awareness of pre-conception planning for women at risk of perinatal mental illness

- Understand the need for lower thresholds for intervention in perinatal populations

- Appreciate the particular characteristics in risk assessment in perinatal populations, taking into account the impact of perinatal psychiatric disorder on both mother and baby

\section{DECLARATION OF INTEREST}

None

The Perinatal Section of the UK's Royal College of Psychiatrists came into being in 1996 and became a Faculty in June 2014. Given that all psychiatric illnesses affecting women of childbearing potential can occur during the perinatal period, there is still some controversy surrounding the way in which services should be commissioned and delivered. General adult and liaison psychiatrists in the UK have always treated patients in the perinatal period. However, the independent report following the deaths of Dr Daksha Emson, a consultant psychiatrist, and her 3-month-old baby during a psychotic episode in 2000 (North East London
Strategic Health Authority 2003) was one of a number of contributors to increased government attention to the needs of this population.

Descriptions of mental illness apparently specific to the perinatal period can be traced back to the first treatise on postnatal illness, written by Louis Victor Marcé, a student of Esquirol (Marcé 1858). Today, there is a growing epidemiological and neurophysiological evidence base showing that underidentification of mental illness in the perinatal period and ineffective treatments can result in both short- and long-term risks to the mother and infant. There is therefore a rationale for increased attention and resources to be directed to this rapidly expanding specialty, but there continues to be widely varying and inequitable distribution of perinatal mental health services in the UK and globally (Almond 2009).

It is not our intention to describe specific clinical conditions. Instead, we will highlight the differences that are associated with presentation in the perinatal period and the management of women during this time. We hope to clarify the value of specialised services in general settings and improve the interface with all clinicians who currently provide care to women and their families.

\section{Current service provision}

In England and Wales, the Health and Social Care Act 2012 reinforced the need for the National Health Service (NHS) to encompass preventive as well as restorative elements. The public health agenda fits neatly alongside perinatal mental health service provision (Department of Health 2011), and health and wellbeing boards are additionally well placed to oversee service provision. However, access to specialised perinatal services remains inconsistent across the UK, as service development continues to be piecemeal and unfortunately all too often driven by serious untoward incidents.

Quality standards defining criteria for accreditation of acceptable community perinatal services were published by the Royal College of Psychiatrists in 2014 (Thompson 2014a) and these have helped generate maps that show large areas of the UK with either very limited perinatal services or no provision at all (Everyone's Business 2014).
Jacqueline Humphreys is a consultant perinatal psychiatrist with East London NHS Foundation Trust. Janet Obeney-Williams is a Year 5 specialist trainee (ST5) in general adult psychiatry with Camden and Islington NHS Foundation Trust. Rachael W. Cheung is an MSc student in developmental neuroscience and psychopathology at University College London. Nisha Shah is a consultant perinatal psychiatrist with North East London NHS Foundation Trust. Correspondence Dr Jacqueline Humphreys, East London NHS Foundation Trust, Perinatal Mental Health Team, Newham University Hospital, Glen Road, London E13 8SL, UK. Email: jacqueline. humphreys@elft.nhs.uk 
MBRRACE-UK: Mothers and Babies: Reducing Risk through Audits and Confidential Enquiries across the UK.
These gaps in service delivery are currently being filled by practitioners within stretched general adult and liaison services.

In-patient mother and baby units are also inequitably distributed across the UK (Hogg 2012), and there are insufficient beds for needs predicted by epidemiological surveys of perinatal mental illness (Joint Commissioning Panel for Mental Health 2012). Travelling a long distance for a mother and baby unit bed will mean that a woman can remain with her baby, but at the expense of being separated from her family and social support network, with potential adverse consequences.

Development of perinatal services is supported by economic modelling, which can help to identify areas where savings can be made (Bauer 2014). Savings could be made not only for healthcare systems, but also for Social Services, which are often involved in supervision of children of mothers requiring mental healthcare (Howard 2003). For example, specialist perinatal services can help identify predictors of outcome after discharge from mother and baby units, thus targeting subsequent social care more efficiently.

Properly commissioned community services integrate parent-infant care with adult mental healthcare. One such service works with parents and children up to 3 years of age and provides joint care with maternity services, home visits and a multiprofessional team that includes midwives. The aim is to intervene at the earliest possible opportunity by close collaboration between acute healthcare providers (including a joint obstetric clinic), primary care, mental health and psychotherapy services. The drive to meet accreditation standards promotes service development.

The number of interfaces between a perinatal psychiatric service and other social and healthcare services is at least double that in other psychiatric settings. The patients have complex needs anyway (as a colleague once commented, birth is a messy business!), and presentations can be affected by the large number of factors that have to be taken into account. For example, the fear of abandonment experienced by women with borderline personality disorder might be reduced during pregnancy if attachment develops with the unborn. However, as the child becomes more independent this can have an impact on the woman's mental health, and targeted interventions can address potential difficulties. Relationships with Social Services can be challenging in all psychiatric specialties, but specific training to enable consideration of the human right of women with mental illness to be mothers is a necessary part of service provision in a perinatal setting.

\section{Training}

The provision of training in perinatal psychiatry remains a challenge for all specialties working with pregnant women. In particular, the most recent MBRRACE-UK ${ }^{a}$ report (Knight 2015) highlighted the need for additional education for liaison and crisis/home treatment teams in the features and risks of perinatal mental illness, given that they often provide emergency and out-of-hours care to these women.

There is also a need for training for general practitioners (GPs), obstetricians, midwives and health visitors, as they are vital in identifying emerging perinatal mental illness in pregnant women and new mothers. The gap in knowledge in GPs was identified in a recent survey in which $40 \%$ of GPs questioned said that they had never received any specific training in perinatal mental health (Khan 2015). Meanwhile, the government's mandate to Health Education England (HEE) requires HEE to ensure the development of training in perinatal mental illness for both trainee and qualified midwives (Department of Health 2014), as well as commissioning further work with the Royal Colleges of General Practitioners, Obstetricians and Psychiatrists to incorporate perinatal mental health into their postgraduate syllabuses.

In 2010, the Perinatal Section of the Royal College of Psychiatrists drew up a draft competency based curriculum for specialists in perinatal psychiatry (Royal College of Psychiatrists 2010). In September 2016, this is still awaiting approval by the General Medical Council, so at present specialist endorsement for perinatal psychiatry in the UK does not exist. In the meantime, the provision of training for psychiatrists remains a priority for the Perinatal Faculty to address.

The setting up of regional perinatal networks can be a key driver in the development and implementation of training schemes across specialties.

\section{Political context}

Like all psychiatric specialties, perinatal psychiatry has a political context. The impact of gender issues on the management of perinatal mental illness cannot be understated and there is an argument that gender politics have influenced provision of services historically.

The transition from being in full-time employment to becoming a dedicated mother can result in both financial pressure and social isolation. It has been well established that single mothers with reduced income and poor social support are at higher risk of developing depression (Brown 1997). 
This effect is more marked in women in lowand middle-income countries (Halbreich 2006), where there are fewer resources for treatment (Almond 2009). Factors related to socioeconomic disadvantage are often determinants of depression. These include the young age of mothers, being unmarried, hostility from in-laws, violence from the partner, lack of support, previous mental illness and, sometimes, giving birth to a girl. A higher than average level of education, permanent employment, being from an ethnic majority and having a kind, trustworthy partner have been identified as protective factors (Fisher 2012).

In addition to dealing with their own mental health needs, women must also contend with the demands of being a parent, both for their new baby and for other children in the household. This caregiving role can increase the pressure to recover more quickly, and to a higher level of functioning, than in other psychiatric populations.

For some women, prioritising their children's needs above their own may result in reduced concordance with medication, especially if it is sedative (Nicholson 1998).

There is increasing interest in partner violence during pregnancy (Jasinski 2004) and its relationship to perinatal mental illness, as it significantly increases the odds of high levels of depressive symptoms as well as anxiety and posttraumatic stress disorder (Howard 2013).

\section{Paternal mental illness}

The impact of mental illness in the father merits more attention, and paternal mental illness is specifically addressed in some perinatal services. For example, family and couples therapy is listed as an accreditable service standard for perinatal community mental health services (Thompson 2014a). In April 2015, new legislation in England introduced shared parental leave (SPL) and statutory shared parental pay (ShPP). These enable parents to split up to 50 weeks of leave following the initial 2 weeks given to mothers after the birth (UK Government 2015). Thus, mothers may return to work sooner, leaving the father in charge of caring for the new baby. The effect of this change in the law on paternal mental illness remains to be seen.

\section{Stigma}

The stigma associated with psychiatric illness can prevent individuals from seeking help (Rüsch 2005). There are particular issues associated with mothering and mental illness - related to society's view of what is required to care for a baby. These cultural attitudes may be attributed in part to our understanding of maternal influence on children's development gained from academics such as Winnicott (1953), and are likely also influenced by the media. One online survey of 1250 mothers reported that half of those who had completed the Edinburgh Postnatal Depression Scale gave false information in an attempt to conceal their difficulties, for reasons such as fear of being branded a 'failure' as a mother (Russell 2006). Mothers with mental illness can feel pressure to prove that they are able to meet their child's needs, and may be unwilling to rely heavily on their partner or other family members. These feelings can be magnified by fears that the parenting role will be disrupted (for example, that the child will be taken into care) if mental illness is identified (Nicholson 1998) and reinforced by professionals' need to consider safeguarding and sometimes the involvement of Social Services to provide essential support.

Celebrity culture and the media have helped to promote positive images of perinatal mental illness. Celebrities such as Brooke Shields and Marie Osmond, for example, have written books detailing their struggles with postpartum depression. It is possible that, similar to the so-called Jade Goody effect in relation to cervical screening (Lancucki 2012), these accounts might encourage patients to defy stigma and alert professionals to perinatal psychiatric problems.

However, there is the potential risk that media reports of mainly severe and often fatal perinatal psychiatric disorders such as those experienced by Charlotte Bevan and Felicia Boots may prevent other women coming forward owing to fear of association.

Specialised perinatal psychiatric services can actively combat stigma by provision of clinics in maternity settings, incorporation of specialised midwives into their teams and active engagement with acute and primary care colleagues, including the provision of training programmes.

\section{Clinical presentation}

The clinical presentation of women to a perinatal psychiatric service varies widely. At one end of the spectrum are individuals who are currently well but at risk of becoming unwell because of a history of mental illness. At the other end, there are individuals with the rapidly evolving and serious presentation of puerperal psychosis.

Postnatal depression has been extensively researched, but it is now acknowledged that depression often arises in the antenatal period (Howard 2014). Increasing attention is being paid to anxiety disorders such as obsessive-compulsive 
disorder and post-traumatic stress disorder, which can emerge for the first time in the perinatal period, with specific features associated with childbirth and parenting (Ayers 2004; Russell 2013). In addition, services must cater for women with pre-existing mental illness, including psychotic illness, eating disorders and personality disorders. Women with bipolar affective disorder have a 30$70 \%$ chance of relapse after giving birth (Viguera 2007). The risk of relapse of schizophrenia after delivery is not so high, but there can be other challenges, such as promoting mother and baby interaction in the presence of ongoing negative symptoms (Snellen 1999).

To better understand the care of women in the perinatal period, it is important to consider the physiological and psychological changes of normal pregnancy, labour and child-rearing. Women commonly experience mood swings, sleep disruption and increased fatigue, both during pregnancy (especially the first and third trimesters) and in the postnatal period. Increased levels of anxiety are well recognised, especially in first-time mothers. A psychiatric service dedicated to pregnant women that has close links with professionals such as midwives and obstetricians can distinguish between the normally observed and the pathological.

\section{Thresholds}

Since the closure of the asylums first began, government legislation, public opinion and economic considerations have been driving a general trend towards fewer psychiatric in-patient beds in favour of community care (Killaspy 2006). As the caseloads of community psychiatric services have grown, the thresholds for accessing specialised mental health services have been raised, and inpatient beds are now mainly for those with severe conditions such as psychosis. However, many women at risk of maternal mental illness present well at the time of first booking and during their pregnancy. Generic psychiatric services can find it difficult to provide support for this cohort, who on initial assessment appear more appropriate for primary care, but who nevertheless often require active management to prevent relapse during the antenatal and postnatal periods.

In its latest report on maternal deaths in the UK (Knight 2015), MBRRACE-UK has identified three red-flag signs that indicate the possibility of serious maternal mental illness (Box 1) and also symptoms that should prompt consideration for admission to a mother and baby unit (Box 2).

For women presenting with active symptoms of mental illness, it is important that thresholds
BOX 1 Red-flag signs of serious maternal mental illness

The following indicate need for urgent senior psychiatric assessment:

- recent significant change in mental state or emergence of new symptoms

- new thoughts or acts of violent self-harm

- new and persistent expressions of incompetency as a mother or estrangement from the infant

(Knight 2015: p. 32)

are adjusted to incorporate the needs of the child. These women require a much higher level of functioning to perform their caring role both for their baby and their other children. For example, a mother with obsessive-compulsive disorder and fears of contamination may struggle with preparing bottles and changing nappies (Box 3). In fact, any obsessional or compulsive symptoms can have a significant impact on caring for a baby and on the mother-infant relationship.

\section{Management}

Decision-making

Treatment decisions are particularly complex in the perinatal period. Involving women in the decision-making process is crucial to the provision of quality care (Patel 2011). There are numerous dilemmas to be faced by women and clinicians, from planning pregnancies to the treatment of illness, and managing both new and relapsing conditions. General principles of prescribing should be adhered to as with a non-perinatal population: maximising non-drug treatments and avoiding polypharmacy where possible, while also avoiding under-treatment and potential relapse that might necessitate greater total doses of medication to regain symptom control.

BOX 2 Admission to a mother and baby unit

Admission to a mother and baby unit should always be considered if the woman has any of the following:

- rapidly changing mental state

- suicidal ideation (particularly of a violent nature)

- pervasive guilt or hopelessness

- significant estrangement from the infant

- new or persistent beliefs of inadequacy as a mother

- evidence of psychosis (Knight 2015: p. 36) 


\section{Pre-conception and birth planning}

Specialised assessment is key for prevention of poor outcomes in perinatal psychiatry. For women with a psychiatric history, pre-conception meetings, for example, can enable careful planning for the perinatal period and address matters such as when to get pregnant, what medications to use and what to expect (Box 4). ${ }^{\mathrm{b}}$ It is important to remember that unplanned pregnancies are more common in women with a diagnosis of mental illness (Hall 2014).

The latest update of National Institute for Health and Care Excellence (NICE) guidelines on antenatal and postnatal mental illness re-emphasises the need to discuss the possibility of pregnancy with all women of childbearing potential taking psychotropic medication (National Institute for Health and Care Excellence 2014). This will include possible avoidance of medications that can reduce fertility, such as prolactin-elevating antipsychotics.

Pre-birth planning at 32 weeks' gestation is advisable for women with severe and enduring mental illness. These can be run as a care programme approach (CPA) meeting, with the woman inviting all involved health professionals and supportive significant others. Social Services may be present with the patient's explicit consent. Plans are formulated for monitoring and management during delivery and the postnatal period. Specific considerations include the woman's relapse triggers and indicators, allocation of a side-room to maximise sleep, stopping or changing medication such as lithium, assessment after delivery, and whether the support of her local crisis team is likely to be sufficient in an emergency or whether admission to a mother and baby unit should be planned. Plans for sleep protection can be made for once the woman goes home, taking into account partners, family members or professional help. Prophylactic admission to a mother and baby unit (i.e. prior to delivery) may be recommended if the chance of relapse is thought to be very high (Box 5).

\section{Medication}

All women of childbearing age taking psychotropics should have access to information on the drugs' potential effects on pregnancy. As psychotropics cross the blood-brain barrier, they tend also to be readily transported across the placenta (Källén 2013). Any drug prescribed may pose theoretical risks to the unborn child, and for many women any risk to the infant is unacceptable.

Some drugs are known to have increased risk of teratogenesis. For example, it is now well

BOX 3 Case vignette: how preventive work can influence outcomes

Anna is 28 years old and in the 35 th week of pregancy. She is known to the perinatal psychiatric service owing to exacerbation of obsessive-compulsive disorder after two previous births.

She was seen in clinic with her mother. She gave a history of checking her daughter's underwear for maggots each morning. She was sleeping poorly, having trouble with eating and drinking and had cognitive features of depression. She is receiving psychological therapy and considering taking an SSRI after the birth.

Anna invited her partner and her mother to the pre-birth planning meeting, but not the child and family social worker. There is to be a discharge planning meeting after the birth, convened by Social Services. She also has follow-up in clinic in a month's time, with access to care if needed before then. It has been carefully explained to her and her family that, should the situation deteriorate, in particular with regard to her nutritional status, she can be admitted to hospital under perinatal services.

This case shows the importance of preventive work in perinatal psychiatry. The right treatment at the right time can prevent adverse outcomes and ensure that safeguards are adequately placed. Consideration of risks to other family members is part of the management plan.

established that sodium valproate has teratogenic effects on the developing fetus, and the latest NICE guidelines (2014) recommend that no woman of childbearing potential be prescribed sodium valproate unless there is no alternative. Other known teratogens include carbamazepine. However, for many medications very little is known owing to a lack of evidence. Familiarity with the rapidly evolving evidence base is therefore required.

When discussing medication with women, the risks of use must be weighed against risks of discontinuation. It is important to consider the risks of untreated illness, and this needs to be tailored to each individual patient. In addition to potential relapse, other consequences need to

\section{BOX 4 Case vignette: pre-conception counselling}

Rosaline, a 24-year-old woman living in London, was referred to the perinatal psychiatric service by her general adult psychiatrist. Originally from Greece, she was diagnosed there with bipolar disorder, schizophrenia and schizoaffective disorder. She has a family history of bipolar disorder and when unwell she becomes a danger to both herself and others. She has made two previous suicide attempts, both of which resulted in hospital admission in Greece. She has been treated in the past with sodium valproate, lithium and olanzapine.

She is being treated with olanzapine in the UK, and wants to become pregnant with her partner here. However, she has family problems requiring her to return to Greece b. All case vignettes in this article are fictitious. . 
BOX 5 Case vignette: admission thresholds

Polly is 34 years old and has a diagnosis of emotionally unstable personality disorder and previous psychotic symptoms. She was referred at 26 weeks' gestation by her general practitioner to the community psychiatric assessment service. However, she did not attend her assessment appointment and so was discharged back to primary care. Four days after the birth, Polly drank a bottle of champagne and became elated in mood. Her family brought her to the emergency department, where she was assessed by the crisis team. She insisted she was well enough to take care of the baby and would not be drinking again. Therefore they decided she did not meet the threshold for crisis team involvement.
Her family brought her in again after the weekend with increasing symptoms of bizarre behaviour. She was seen by the perinatal psychiatric team and referred back to the crisis team, who this time accepted the referral. It soon became clear that she needed admission, and she was admitted for treatment to the mother and baby unit, where she stayed for 4 months and her diagnosis was revised to include bipolar affective disorder.

In this situation, with a history of mood instability, earlier referral to perinatal services might have better contained the risk. Specialist perinatal services have lower thresholds for assessment and treatment, because of the potential risk of harm to both mother and baby.

be considered. For example, studies have found that rates of preterm birth are increased both for pregnant women taking selective serotonin reuptake inhibitors (SSRIs) and for those whose depression is untreated (Wisner 2009).

Medication levels are influenced by the physiological changes in pregnancy and the postnatal period (Box 6). This is important to bear in mind when considering minimum effective doses and toxicity levels. A well-known example is lithium, when rapid dose adjustment is needed at the time of labour.

Breastfeeding decisions require up-to-date information on medications, as all drugs transfer into human milk to some degree, although this rarely results in clinically significant doses for the infant. Relative infant dose can be a helpful

BOX 6 Physiological effects of pregnancy relating to drug metabolism

- Reduced gastric emptying

- Reduced lean body mass

- High cardiac output

- Low plasma protein binding

- Reduced drug-binding capacity (albumin)

- Increased blood and plasma volumes (40-50\%)

- Reduced cholinesterase activity

- Poor hepatic functioning

- Raised renal plasma flow and renal clearance

- Higher permeability of blood-brain barrier

tool to guide these decisions. Factors influencing transfer are maternal plasma concentrations, molecular weight, degree of protein binding and ease of passage across the blood-brain barrier (Hale 2014).

\section{Electroconvulsive treatment}

Further controversy exists concerning the role of electroconvulsive treatment (ECT) in the treatment of perinatal psychosis. Low risk to mother and fetus has previously been described (Anderson 2009). A more recent systematic review of case studies showed that there are limited published studies on how to administer ECT in early pregnancy and revealed an overall child mortality of $7.1 \%$ across case studies (Leiknes 2015). Nevertheless, ECT remains a potentially life-saving option (Howard 2014).

\section{Psychological therapy}

Social factors are clearly stressors in the first postnatal year, with financial problems contributing significantly to risk (Segre 2007). Other risk factors for anxiety and depression include relationship stresses and housing concerns, but these can also be consequences of depressive illness, thus making it difficult to extrapolate cause from reported associations.

Psychological therapies in pregnancy need to tackle specific problems, and have been shown to be effective (Dennis 2007). They must be tailored to achieve best results for a perinatal population; when classic cognitive-behavioural therapy has been used without modification for postnatal depression, results have been mixed (O'Mahen 2012). Recent literature reviews show that most of the evidence regarding psychological therapies is limited to postnatal depression, with fewer studies covering either the antenatal period or other non-psychotic disorders (Howard 2014). Nevertheless, psychological therapy forms a key part of treatment, given the increased risks of medication management and patient choice.

Perinatal psychiatry can facilitate a creative approach to care. Art therapies and nursery nurses are included in Royal College of Psychiatrists accreditation standards (Thompson 2014a,b).

\section{The evidence base}

Randomised controlled trials (RCTs) are far more difficult with pregnant women (Wisner 2012), and newer methods such as patient preference RCTs may enable a greater body of evidence to be gathered (Howard 2006). Specific treatment recommendations tend not to endure as more evidence rapidly accumulates, but the perinatal 
psychiatrist must be able to discuss individualised risk-benefit decisions for the drug treatment of all pregnant and breastfeeding women, based on up-to-date data. Appraisal of evidence is not straightforward, as is it difficult to exclude confounders, including the impact of the illness itself. Outcomes such as preterm birth may be the result of several different mechanisms of action (Yonkers 2012).

Measurement of outcomes must take the wellbeing of both mother and child into consideration. Recovery from mental illness must incorporate evidence of ability to meet the emotional, cognitive and behavioural demands of parenting. Depression may persist beyond the first postnatal year, thus increasing its impact on children and the wider community (McCue Horwitz 2007).

NHS England and Monitor now implement tariffs for national prices for care given by providers (Monitor 2016). This application of tariffs can make outcome measurement in maternal mental health particularly difficult. Perinatal psychiatric care allows many high-risk women to remain well throughout and after their pregnancy. This work is complicated to measure.

Treatment delivered to women with severe mental illness on in-patient mother and baby units has been shown to improve the mother-infant interaction (Kenny 2013). However, we do not have epidemiological evidence of the cost-effectiveness of treating mothers and babies in facilities that admit them together. Such data would undoubtedly help support the increased provision of these units worldwide (Glangeaud-Freudenthal 2014).

Specialist perinatal services can help identify predictors of outcome after admission to mother and baby units, thus targeting subsequent social care more efficiently. Hipwell \& Kumar (1996) found mother-infant interaction scales, specialist nurse ratings and maternal psychiatric diagnoses to be strongly predictive of outcomes for perinatal in-patients. The specialised multidisciplinary team on a specialist psychiatric mother and baby unit enabled allocation of follow-up supervision based on predicted outcomes.

\section{Risk and impact}

It can be easy to focus on the potential risks that treatment such as medication can pose; however, it should be remembered that under-treatment of perinatal mental illness also poses both acute and chronic risks to mother and child.

Suicide has been identified as one of the leading indirect causes of maternal mortality in the UK since 2003. The latest MBRRACE-UK report includes a section examining deaths related to psychiatric illness between 2009 and 2013. It found that 161 women died from psychiatric causes during or up to 1 year after the end of the pregnancy, and that nearly a quarter of 'late' maternal deaths (between 6 weeks and 1 year postpartum) had psychiatric causes (Knight 2015).

In the postpartum period, risks of suicide among women with depression are significantly raised, with suicide accounting for $20 \%$ of postpartum deaths (Lindahl 2005). In addition, suicidal ideation has been found in $9 \%$ of women $6-8$ weeks postpartum; younger age, higher parity and higher levels of depressive symptoms are all associated risk factors (Howard 2011).

Although it remains rare, infanticide is sometimes a tragic consequence of maternal mental illness, occurring most commonly when the mother is suffering from postpartum psychosis. Psychotic symptoms focused on the infant are the most worrying indicator in this situation, and clinicians must enquire closely to establish whether these are present.

Some longer-term risks are particular to single mothers, who are at increased risk of poorer long-term physical and mental health, as are their families (Meltzer-Brody 2014). Antenatal depression is associated with poorer obstetric outcomes (Larsson 2004) and independently associated with offspring depression at 18 years of age, which carries major public health implications worldwide (Pearson 2013). The same association exists postnatally only for women with poor education. Such findings suggest that these groups should be targeted to prevent their problems recurring in the next generation.

Other risks to offspring that have been found in untreated maternal mental illness include reduced birth weight, higher rates of infection and temperamental difficulties (Stein 2014). There are multiple potential reasons for these findings, including various illness-linked behaviours such as smoking and poor diet, which can have a higher prevalence in women with mental illness (Shah 2006). Transmission of prenatal stress is thought to be mediated by the hypothalamic-pituitaryadrenal (HPA) axis in both mother and offspring (O’Donnell 2009) and may influence cognitive development (Bergman 2010).

A relatively new area of research is looking at how maternal health in pregnancy can influence child health through epigenetic mechanisms. This provides evidence that maternal obesity and gestational diabetes can result in lifelong risks for the offspring of obesity, and metabolic and cardiovascular disease (Lehnen 2013). This can be an important consideration if prescribing 
medication that is known to cause weight gain or metabolic syndrome.

In 2014, the economic impact of untreated maternal mental illness in the UK was estimated to be around $£ 8.1$ billion for each 1-year cohort of births; nearly $75 \%$ of this total results from impact on children (Bauer 2014). Of this figure, $£ 1.2$ billion falls to the NHS, while it is estimated that an extra $£ 280$ million a year would be sufficient to improve perinatal mental health services nationwide to the level recommended by the Royal College of Psychiatrists (Thompson 2014a).

\section{Conclusions}

Perinatal mental health services in the UK have been developing rapidly since the inauguration of the Perinatal Section of the Royal College of Psychiatrists in 1996. Despite resource limitations, which have resulted in geographically heterogeneous service provision, much work is currently underway to develop uniformly highquality provision of mental healthcare for this vulnerable population of women and their families.

Care in the perinatal period is complex and influenced by political agendas. Regardless, the range of clinical presentations remains broad, with specific features that would appear to have biological as well as psychosocial contributors.

Evidence to support practice is being gathered in both traditional epidemiological and newer ways. Clinicians practising in a risk-averse culture must stay abreast of new findings, especially with respect to prescribing decisions. Any psychiatrist providing care to women of childbearing potential must balance the risks of treatment against those of under-treatment for both adult patients and unborn or newly born children. These risks can involve outcomes with high societal impact, thus thresholds for involvement of the specialist should be low.

The complexity involved requires specialist perinatal services nationwide. However, it will likely always remain the case that general adult psychiatrists will also be involved in the care of these women. Training is important in learning to recognise the signs and symptoms of maternal mental illness as well as when to seek specialist advice.

Despite all the challenges in the current economic climate, perinatal psychiatry offers a stimulating career working with a population that is, for obvious reasons, full of hope.

\section{References}

Almond P (2009) Postnatal depression: a global public health perspective. Perspectives in Public Health, 129: 221-7.
Anderson EL, Reti IM (2009) ECT in pregnancy: a review of the literature from 1941 to 2007. Psychosomatic Medicine, 71: 235-42.

Ayers S (2004) Delivery as a traumatic event: prevalence, risk factors, screening and treatment. Clinical Obstetrics \& Gynecology, 47: 552-67.

Bauer A, Parsonage M, Knapp M, et al (2014) The Costs of Perinatal Mental Health Problems. London School of Economics and Centre for Mental Health.

Bergman K, Sarkar P, Glover V, et al (2010) Maternal prenatal cortisol and infant cognitive development: moderation by infant-mother attachment. Biological Psychiatry, 67: 1026-32.

Brown G, Moran P (1997) Single mothers, poverty and depression. Psychological Medicine, 27: 21-33.

Dennis CL, Hodnett E (2007) Psychosocial and psychological interventions for treating postpartum depression. Cochrane Database Systematic Review, 4: CD006116

Department of Health (2011) No Health Without Mental Health: A Cross-Government Mental Health Outcomes Strategy for People of all Ages. Department of Health.

Department of Health (2014) Delivering High Quality, Effective, Compassionate Care: Developing the Right People with the Right Skills and the Right Values. A Mandate from the Government to Health Education England: April 2014 to March 2015. Department of Health.

Everyone's Business (2014) UK Specialist Community Perinatal Mental Health Teams (Current Provision). everyonesbusiness.org. uk (http://everyonesbusiness.org.uk/wp-content/uploads/2014/07/ UK-Specialist-Community-Perinatal-Mental-Health-Teams-currentprovision.pdf)

Fisher J, Cabral de Mello M, Patel V, et al (2012) Prevalence and determinants of common perinatal mental disorders in women in lowand lower-middle-income countries: a systematic review. Bulletin of the World Health Organization, 90: 139G-49G.

Glangeaud-Freudenthal NMC, Howard LM, Sutter-Dallay AL (2014) Treatment: mother-infant inpatient units. Best Practice \& Research Clinical Obstetrics \& Gynaecology, 28: 147-57.

Halbreich U, Karkun S (2006) Cross-cultural and social diversity of prevalence of postpartum depression and depressive symptoms. Journal of Affective Disorders, 91: 97-111.

Hale T (2014) Medications and Mothers' Milk (16th edn). Hale Publishing.

Hall KS, Kusunoki Y, Gatny H, Barber J (2014) The risk of unintended pregnancy among young women with mental health symptoms. Social Science \& Medicine, 100: 62-71.

Hipwell AE, Kumar R (1996) Maternal psychopathology and prediction of outcome based on mother-infant interaction ratings (BMIS). British Journal of Psychiatry, 169: 655-61.

Hogg S (2012) Prevention in Mind. All Babies Count: Spotlight on Perinatal Mental Health. NSPCC.

Howard L, Shah N, Salmon M, et al (2003) Predictors of social services supervision of babies of mothers with mental illness after admission to a psychiatric mother and baby unit. Social Psychiatry and Psychiatric Epidemiology, 38: 450-5.

Howard L, Thornicroft G (2006) Patient preference randomised controlled trials in mental health research. British Journal of Psychiatry, 188: 303-4.

Howard LM, Flach C, Mehay A, et al (2011) The prevalence of suicidal ideation identified by the Edinburgh Postnatal Depression Scale in postpartum women in primary care: findings from the RESPOND trial. BMC Pregnancy and Childbirth, 11: 57.

Howard LM, Oram S, Galley H, et al (2013) Domestic violence and perinatal mental disorders: a systematic review and meta-analysis. PLoS Medicine, 10(5): e1001452.

Howard LM, Molyneaux E, Dennis CL, et al (2014) Non-psychotic mental disorders in the perinatal period. Lancet, 384: 1775-88. 
Jasinski JL (2004) Pregnancy and domestic violence: a review of the literature. Trauma, Violence, \& Abuse, 5: 47-64.

Joint Commissioning Panel for Mental Health (2012) Guidance for Commissioners of Perinatal Mental Health Services. Vol. Two: Practical Mental Health Commissioning. JCPMH

Källén B, Borg N, Reis M (2013) The use of central nervous system active drugs during pregnancy. Pharmaceuticals, 6: 1221-86.

Kenny M, Conroy S, Pariante CM, et al (2013) Mother-infant interaction in mother and baby unit patients: before and after treatment. Journal of Psychiatric Research, 47: 1192-8.

Khan L (2015) Falling Through the Gaps: Perinatal Mental Health and General Practice. Centre for Mental Health.

Killaspy $\mathrm{H}$ (2006) From the asylum to community care: learning from experience. British Medical Bulletin, 79: 245-58.

Knight M, Tuffnell D, Kenyon S, et al (eds) (2015) Saving Lives, Improving Mothers' Care: Surveillance of Maternal Deaths in the UK 2011-13 and Lessons Learned to Inform Maternity Care from the UK and Ireland Confidential Enquiries into Maternal Deaths and Morbidity 2009-13. National Perinatal Epidemiology Unit, University of Oxford.

Lancucki L, Sasieni P, Patnick J, et al (2012) The impact of Jade Goody's diagnosis and death on the NHS Cervical Screening Programme. Journal of Medical Screening, 19: 89-93.

Larsson C, Sydsjö G, Josefsson A (2004) Health, sociodemographic data, and pregnancy outcome in women with antepartum depressive symptoms. Obstetrics \& Gynecology, 104: 459-66.

Lehnen H, Zechner U, Haaf T (2013) Epigenetics of gestational diabetes mellitus and offspring health: the time for action is in early stages of life. Molecular Human Reproduction, 19: 415-22.

Leiknes KA, Cooke MJ, Jarosch-von Schweder L, et al (2015) Electroconvulsive therapy during pregnancy: a systematic review of case studies. Archives of Women's Mental Health, 18: 1-39.

Lindahl V, Pearson L, Colpe L (2005) Prevalence of suicidality during pregnancy and the postpartum. Archives of Women's Mental Health, 8: 77-87.

Marcé LV (1858) Traité de la folie des femmes enceintes des nouvelles accouchées et des nourrices et considérations médico-légales qui se rattachent à ce sujet [Treatise on Madness in Pregnant Women, in Women who Have Recently Given Birth, and in Wet Nurses, and Medical/Legal Considerations on this Subject]. JB Baillière et fils.

McCue Horwitz S, Briggs-Gowan MJ, Storfer-Isser A, et al (2007) Prevalence, correlates, and persistence of maternal depression. Journal of Women's Health, 16: 678-91.

Meltzer-Brody S, Stuebe A (2014) The long-term psychiatric and medical prognosis of perinatal mental illness. Best Practice \& Research: Clinical Obstetrics \& Gynaecology, 28: 49-60.

Monitor, NHS England (2016) 2016/2017 National Payment System. Monitor (https://www.gov.uk/government/uploads/system/uploads/ attachment_data/file/509697/2016-17_National_Tariff_Payment_ System.pdf).

National Institute for Health and Care Excellence (2014) Antenatal and Postnatal Mental Health: Clinical Management and Service Guidance (Clinical Guidance CG192) NICE.

Nicholson J, Sweeney EM, Geller JL (1998) Focus on women: mothers with mental illness: I. The competing demands of parenting and living with mental illness. Psychiatric Services, 49: 635-42.

North East London Strategic Health Authority (2003) Report of an Independent Inquiry into the Care and Treatment of Daksha Emson M.B.B.S. MRCPsych, MSC. and her Daughter Freya. North East London Strategic Health Authority (http://www.simplypsychiatry.co.uk/ sitebuildercontent/sitebuilderfiles/deinquiryreport.pdf).

O'Donnell K, O'Connor TG, Glover V (2009) Prenatal stress and neurodevelopment of the child: focus on the HPA axis and role of the placenta. Developmental Neuroscience, 31: 285-92.
O'Mahen H, Fedock G, Henshaw E, et al (2012) Modifying CBT for perinatal depression: what do women want? A qualitative study. Cognitive and Behavioral Practice, 19: 359-71.

Patel SR, Wisner KL (2011) Decision making for depression treatment during pregnancy and the postpartum period. Depression and Anxiety, 28. 589-95

Pearson RM, Evans J, Kounali D, et al (2013) Maternal depression during pregnancy and the postnatal period: risks and possible mechanisms for offspring depression at age 18 years. JAMA Psychiatry, 70: 1312-9.

Royal College of Psychiatrists (2010) A Draft Competency Based Curriculum for Specialist Training in Psychiatry: Specialist in General Psychiatry with Sub-Specialty in Perinatal Psychiatry. Royal College of Psychiatrists (http://www.rcpsych.ac.uk/pdf/ PerinatalPsychiatryDraftCurriculumNovember2010.pdf).

Rüsch N, Angermeyer AC, Corrigan PW (2005) Mental illness stigma: concepts, consequences, and initiatives to reduce stigma. European Psychiatry, 20: 529-39.

Russell S (2006) Barriers to care in postnatal depression. Community Practitioner, 79: 110-1.

Russell J, Fawcett JM, Mazmanian D (2013) Risk of obsessivecompulsive disorder in pregnant and postpartum women: a metaanalysis. Journal of Clinical Psychiatry, 74: 377-85

Segre LS, O'Hara MW, Arndt S, et al (2007) The prevalence of postpartum depression. Social Psychiatry and Psychiatric Epidemiology, 42: $316-21$.

Shah N, Howard L (2006) Screening for smoking and substance misuse in pregnant women with mental illness. The Psychiatrist, 30 : $294-7$.

Snellen M, Mack K, Trauer T (1999) Schizophrenia, mental state, and mother-infant interaction: examining the relationship. Australian and New Zealand Journal of Psychiatry, 33: 902-11.

Stein A, Pearson RM, Goodman S, et al (2014) Effects of perinatal mental disorders on the fetus and child. Lancet, 384: 1800-19.

Thompson P, Rodell H (eds) (2014a) Service Standards: Second Edition. Perinatal Community Mental Health Services (CCOI171). Royal College of Psychiatrists, College Centre for Quality Improvement (http:// www.rcpsych.ac.uk/pdf/Perinatal\%20Comunity $\% 20$ Standards $\% 20$ Cycle\%203.pdf).

Thompson P, Mahon K (eds) (2014b) Service Standards for Mother and Baby Units: Fourth Edition (CCOI161). Royal College of Psychiatrists, College Centre for Quality Improvement (http://www.rcpsych.ac.uk/ pdf/Perinatal\%20lnpatient\%20Standards\%20Cycle\%208.pdf).

UK Government (2015) Shared Parental Leave and Pay. GOV.UK (https://www.gov.uk/shared-parental-leave-and-pay/overview). Accessed 23 June 2016

Viguera AC, Whitfield T, Baldessarini RJ, et al (2007) Risk of recurrence in women with bipolar disorder during pregnancy: prospective study of mood stabilizer discontinuation. American Journal of Psychiatry, 164: 1817-24

Winnicott DW (1953) Transitional objects and transitional phenomena - a study of the first not-me possession. International Journal of Psychoanalysis, 34: 89-97.

Wisner KL, Sit DK, Hanusa BH, et al (2009) Major depression and antidepressant treatment: impact on pregnancy and neonatal outcomes. Psychosomatic Medicine, 7: 374-84.

Wisner KL (2012) The last therapeutic orphan: the pregnant woman. American Journal of Psychiatry, 169: 554-6.

Yonkers KA, Norwitz ER, Smith MV, et al (2012) Depression and serotonin reuptake inhibitor treatment as risk factors for preterm birth. Epidemiology, 23: 677-85.

\section{MCO answers}

1 a 2 e 3 c $\quad 4$ b 5 e 


\section{MCOs}

Select the single best option for each question stem

1 In a perinatal psychiatric risk assessment, the clinician should be most concerned by: a a delusion that the baby has 'demon eyes'

$\mathrm{b}$ a family history of puerperal psychosis

c ongoing arm cutting

d affective blunting

e current methadone use.

2 In the assessment of perinatal obsessivecompulsive disorder, symptoms that can have a significant impact on the baby are:

a fear of contamination

b rituals involving counting c anxiety regarding involvement of social services

d rumination regarding relationship with baby

e all of the above.

3 Postpartum psychosis differs from presentation at other times in that:

a it takes more time to evolve

b there is less of an association with drugs

c it takes less time to evolve

$\mathrm{d}$ there is a strong affective component

e there is rapid fluctuation of symptoms.

4 Pre-birth planning meetings do not necessarily involve:

a the patient b the child and family social worker

c the midwife

$\mathrm{d}$ the psychiatrist

e the care coordinator.

5 Perinatal patients accepted for crisis or in-patient treatment include:

a women experiencing a psychotic episode

b recently delivered women with a history of postnatal psychosis

c women with postnatal exacerbation of symptoms of obsessive-compulsive disorder

d women with emotionally unstable personality disorder who have thoughts of harm to the self/ baby

e all of the above. 